Title:

\title{
A LES-CMC formulation for premixed flames including differential diffusion
}

Authors:

Daniele Farrace ${ }^{\mathrm{a}, 1}$, Kyoungseoun Chung ${ }^{\text {a }}$, Michele Bolla ${ }^{\mathrm{a}}$, Yuri M. Wright ${ }^{\text {a }}$, Konstantinos Boulouchos ${ }^{\text {a }}$, Epaminondas Mastorakos ${ }^{b}$

a Aerothermochemistry and Combustion Systems Laboratory, Swiss Federal Institute of Technology, Zurich, Switzerland

${ }^{\mathrm{b}}$ Department of Engineering, University of Cambridge, Cambridge, United Kingdom

${ }^{1}$ Corresponding author:

ETH Zurich

Institute of Energy Technology

Aerothermochemistry and Combustion Systems Laboratory

ML L20

Sonneggstrasse 3

CH-8092 Zurich

Switzerland

email: dfarrace@lav.mavt.ethz.ch 


\section{Abstract}

A finite volume Large Eddy Simulation-Conditional Moment Closure (LES-CMC) numerical framework for premixed combustion developed in a previous study [Farrace et al., Proc. Combust. Inst. 36 (2017)], has been extended to account for differential diffusion. The non-unity Lewis number CMC transport equation has an additional convective term in sample space proportional to the conditional diffusion of the progress variable, that in turn accounts for diffusion normal to the flame front and curvature-induced effects. Planar laminar simulations are first performed using a spatiallyhomogeneous non-unity Lewis number CMC formulation and validated against physical-space fully-resolved reference solutions. The same CMC formulation is subsequently used to numerically investigate the effects of curvature for laminar flames having different effective Lewis numbers: a lean methane-air flame with $L e_{e f f}=0.99$ and a lean hydrogen-air flame with $L e_{\text {eff }}=0.33$. Results suggest that curvature does not affect the conditional heat release if the effective Lewis number tends to unity, so that curvature-induced transport may be neglected. Finally, the effect of turbulence on the flame structure is qualitatively analysed using LES-CMC simulations with and without differential diffusion for a turbulent premixed bluff body methane-air flame exhibiting local extinction behaviour. Overall, both the unity and the non-unity computations predict the characteristic "M-shaped" flame observed experimentally, although some minor differences are identified. The findings suggest that for the high Karlovitz number (from 1 to 10) flame considered, turbulent mixing within the flame weakens the differential transport contribution by reducing the conditional scalar dissipation rate and accordingly the conditional diffusion of the progress variable.

Keywords: Large Eddy Simulation; Conditional Moment Closure; Turbulent premixed flames; Differential diffusion

\section{Introduction}

Lean premixed combustion has the potential to achieve the emission limits dictated by stringent legislations, making this strategy desirable for many practical applications [1], e.g. gas turbines or lean burn gas engines. However, while lean mixtures ensure low emission levels, they are susceptible to low Damköhler number phenomena such as local or global extinction [1-3]. The stabilization and blow-off mechanisms of such flames are dictated by complex multi-scale interactions between turbulence and chemical kinetics that influence the flame inner structure depending on the aerothermo-chemical properties of the flow. A comprehensive understanding of these interactions is therefore of supreme importance to define the operability map as well as the emissions of the burner. In this context, numerous experiments 
have been conducted to investigate the influence of turbulence on the flame structure. In [4-6], Gülder and colleagues measured the thermal flame front thickness and the flame turbulent burning velocity of methane-air flames at intense levels of turbulence $\left(u^{\prime} / s_{L}\right.$ up to 24 and $l_{I} /_{L}$ up to 46) using planar Rayleigh scattering and PIV techniques and investigated the effects of turbulent intensity, equivalence ratio and bulk flow velocity. Recently, burning velocity measurements in lean methane-air flames have been extended in [7] to extreme levels of turbulence $\left(u^{\prime} / s_{L}\right.$ up to 163 and $l_{I} /_{L}$ up to 114) where $\mathrm{OH}$ - and $\mathrm{CH}_{2} \mathrm{O}$-PLIF images provided an instantaneous characterization of the flame structure. In [8-11], simultaneous multi-scalar imaging combined with Rayleigh scattering thermometry provided a measure for the flame preheat $\left(\mathrm{CH}_{2} \mathrm{O}\right)$, reaction $(\mathrm{HCO}, \mathrm{CH})$ and oxidation $(\mathrm{OH})$ zone of methane-air flames in the thin and distributed reaction zone regimes. Most of these experimental studies have not focused in detail on non-unity Lewis number flames, however multi-scalar measurements of major species performed in [12] revealed that atomic mass fractions across a bluff body stabilised lean methane-air flame were affected by preferential transport. This suggests that this phenomenon may be important to study in the context of flames stabilised by recirculation zones.

Supporting experimental investigations, Large Eddy Simulation (LES) coupled with state-of-the-art combustion models has emerged as a promising numerical framework [13] to accurately compute non-measurable quantities at practical computational costs. However, the strong interaction among turbulence, chemical reaction and diffusion poses a notable challenge for combustion modelling [14,15]: firstly, the highly non-linear temperature dependence of the reaction rate leads to significant errors when averaged temperatures are considered [16] and secondly, species preferential transport has been observed to be non-negligible, especially at lower turbulent Reynolds numbers [17].

The non-linearity problem is well addressed by both flamelet- and probability-based approaches by decoupling complex chemistry and molecular transport from the turbulent flow field [18]. Flamelet-based methods are typically divided in unstrained flamelets [19-25] where the laminar flamelet is assumed to be thinner than all the turbulent structures and in strained flamelets [26-31] where the contribution of turbulent eddies and hydrodynamic strain is usually included in the formulation of the mean reaction rate. Stochastic methods include the transported probability density function (TPDF) [32-34] where the term related to chemical reaction appears in closed form as convection in scalar space and conditional moment closure (CMC) where chemistry is usually closed at first order exploiting the strong correlation with the conditioning variable [35]. Although CMC has been extensively applied to non-premixed combustion [36,37], only few studies for premixed flames exist and mainly limited to either the RANS context [16,38] or a priori DNS analysis [39]. Recently, a finite volume LES-CMC formulation has been developed and validated for premixed bluff body flames close to blow-off using closures associated with subgrid scale combustion, turbulence and molecular diffusion processes of premixed flames without considering differential diffusion effects [40]. 
However, studies on differential transport increased enormously only in the last decade, reflecting the interests in the development of burners capable of stably burning lean hydrogen mixtures [41]. Several DNS studies with detailed chemistry contributed to a better understanding of the cellular burning behaviour of lean hydrogen flames [42-45]. The observed influence of differential transport on the burning velocity encouraged the extension of such studies over a wide range of more realistic Karlovitz numbers and to heavier fuels including methane [46-48], propane [46] and heptane [49,50]. In general, differential diffusion has been noted to affect species mass fractions by different amounts, depending on the species Lewis numbers [47]. Nevertheless, the overall influence on the flame burning velocity is better described using a mixture effective Lewis number, e.g. as proposed in [51], where a weighted average of the Lewis numbers of the two reactants is proposed based on asymptotic theory of premixed flames. Moreover, turbulence has been observed to attenuate the contribution of differential diffusion [47], necessitating the introduction of a species effective Lewis number (not to be confused with mixture effective Lewis number), $L e_{i, e f f}=\left(+D_{t u r b}\right) /\left(D_{i}+D_{\text {turb }}\right)$, that tends to unity as the turbulence intensity increases [52].

The description of these complex mechanisms in state-of-the-art combustion models such as CMC is not straightforward, though numerous successful works have been already realized in the context of non-premixed combustion using TPDF [53], flamelets [54-56] and CMC [57-59]. Exploiting this knowledge, several methodologies to account for differential diffusion have been extended also to premixed combustion: in [60], a composition-space solution has been derived accounting for a differential diffusion drift between chemical species and composition-space coordinates and good agreement with the physical-space reference solution is reported. A different approach has been adopted in [61], where a two-equation model (progress variable and mixture fraction) is formulated to treat the local equivalence ratio variation across the flame due to non-unity species Lewis numbers. For TPDF, an approach that considers differential diffusion is proposed for pairwise-exchange mixing models and improvements with regards to the unity Lewis number formulation have been observed by comparison with DNS data of turbulent premixed methane-air flames [62]. In the context of RANS-CMC, differential transport is described in the CMC transport equation by an additional term proportional to the conditional diffusion [16,38], where the conditional diffusion is related to the conditional dissipation and the pdf of the progress variable, as derived in [35].

The primary scope of this work is to extend the LES-CMC model first developed in [40] to account for preferential transport using a simple and robust formulation that allows for efficient simulations of close to real-life engines and gasturbines. In order to assess the predictive capability of the model, results computed with the non-unity Lewis number spatially-homogeneous CMC are compared to physical-space fully-resolved reference solutions. The effects of differential diffusion, turbulence and local flame curvature on the flame structure are further evaluated within the CMC 
context. Finally, LES-CMC results with and without differential diffusion are qualitatively discussed through simulations of a turbulent premixed methane-air bluff body flame exhibiting local extinction behaviour.

\section{Modelling}

\subsection{Unstructured LES-CMC for premixed flames}

The CMC methodology is based on the hypothesis that fluctuations of species and temperature are correlated to fluctuations of a representative variable [35], i.e. mixture fraction for non-premixed or progress variable for premixed combustion. While for single-phase flows mixture fraction is a conserved scalar, progress variable is reactive and its transport equation, Eq. (1), contains an additional source term $\widetilde{\dot{w}}$

$$
\frac{\partial \bar{\rho} \tilde{c}}{\partial t}+\nabla \cdot(\bar{\rho} \tilde{\mathbf{u}} \tilde{c})=\nabla \cdot\left[\left(\overline{\rho D}+\bar{\rho} \frac{v_{t}}{S c_{t}}\right) \nabla \tilde{c}\right]+\widetilde{\dot{w}_{c}}
$$

The progress variable is often defined based on fuel mass fraction, temperature or sensible enthalpy [63]. Here, due to the

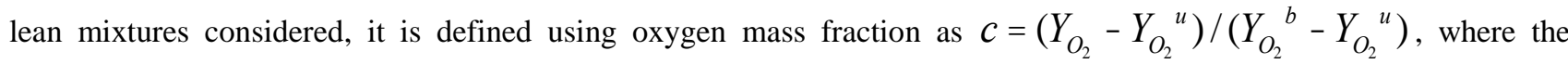
superscripts denote unburnt and burnt states. The reaction rate of Eq. (1), $\widetilde{\dot{w}_{c}}=\widetilde{\dot{w}_{O_{2}}} /\left(Y_{O_{2}}^{b}-Y_{O_{2}}^{u}\right)$, is closed at first order using a detailed combustion mechanism discussed below.

The finite volume formulation of LES-CMC for non-premixed flames has been developed by [37,64]. Here, the finite volume form of the CMC equation for premixed combustion firstly presented in [40] is considered as

$$
\begin{aligned}
& \frac{\partial Q_{i}}{\partial t}+\frac{1}{V} \oint\left(Q_{i} \widetilde{\mathbf{u} \mid \zeta}\right) \cdot d \mathbf{A}-\frac{Q_{i}}{V} \oint(\widetilde{\mathbf{u} \mid \zeta}) \cdot d \mathbf{A} \\
= & \widetilde{N \mid \zeta} \frac{\partial^{2} Q_{i}}{\partial \zeta^{2}}+\widetilde{\dot{w}_{i} \mid \zeta}-\widetilde{\dot{w}_{c} \mid \zeta} \frac{\partial Q_{i}}{\partial \zeta}+E_{f, i}
\end{aligned}
$$

where $Q_{i}=\widetilde{Y_{i} \mid \zeta}$ is the filtered conditional mass fraction and is the sample space variable for $c$. Note that this formulation does not account for differential transport. The second and third terms on the LHS of Eq. (2) represent the decomposition of the convective transport, arising from the finite volume formulation. The first term on the RHS contains the conditional scalar dissipation rate $\widetilde{N \mid \zeta}$, describing molecular mixing. The second and third terms are the species reaction rate and the influence of the conditioning variable on $Q_{i}$. The sub-grid conditional flux 
$E_{f, i}=e_{V} d V$ where $e_{f, i}=-\nabla \cdot\left[\bar{\rho} \tilde{p}(\zeta)\left(\widetilde{\mathbf{u Y} Y_{i} \mid \zeta}-\widetilde{\mathbf{u} \mid \zeta} Q_{i}\right)\right] /(\bar{\rho} \tilde{p}(\zeta))$ is modelled using the simple gradient model [65], leading to $E_{f, i}=1 / V \oint\left(D_{t} \nabla Q_{i}\right) d \mathbf{A}$ where $D_{t}={ }_{t} /{ }^{-} S c_{t}$. This is justified by the negligible contribution of the conditional fluctuation $E_{f, i}$ to the evolution of $Q_{i}$, as discussed in [39].

The conditional velocity is assumed equal to the unconditional value $\widetilde{\mathbf{u} \mid \zeta}=\tilde{\mathbf{u}}$, following successful application to nonpremixed combustion [66]. In premixed configurations it may be justifiable for well-resolved low Damköhler number flames and it has recently been adopted in [40], however its applicability is yet to be validated. The conditional scalar dissipation rate is modelled according to [67] as $\widetilde{N \mid \zeta} \approx \widetilde{N_{c}} \cdot f(\zeta) / \int_{0}^{1} f(\zeta) \tilde{p}(\zeta) d \zeta$, where $f(\quad)$ is the functional dependence on the conditioning variable estimated a priori using laminar flames simulated by PREMIX [68]. This is justified by the weak dependence of $f(\quad)$ on strain rate, especially in the reactive-diffusive layer of the flame [67], and on measurements in low swirl flows [69]. The unconditional scalar dissipation rate, $\widetilde{N}_{c}$, is evaluated at the LES resolution as $\widetilde{N_{c}}=D_{c}(\nabla \tilde{c} \cdot \nabla \tilde{c})+\widetilde{\varepsilon_{c}}$, where $\widetilde{\varepsilon_{c}}$ is the SGS contribution addressed later. Species reaction rates are closed at first order $\widetilde{\dot{w}_{i} \mid \zeta}=\dot{w}\left(Q_{i}, Q_{T}\right)$, following [35]. The filtered conditional averages evaluated at the coarse CMC grid are integrated in each time step on the fine LES grid using a filtered probability density function (FDF), $\tilde{p}(\zeta)$, presumed as a $\beta$-function distribution, as proposed in [70]. This choice is motivated by its ability to provide qualitatively reasonable mono-modal and bimodal pdf shapes at small and large variance values maintaining low computational effort, and by the good predictions reported in previous studies [38,71-76]. In [76], its capability to recover the real pdf for a wide enough range of normalized variances $g=\widetilde{c^{\prime \prime 2}} /(\tilde{c}(1-\tilde{c}))$ is demonstrated. The FDF is calculated at the LES resolution using the filtered progress variable $\tilde{c}$ (Eq. (1)) and the SGS variance $\widetilde{c^{\prime 2}}$. In contrast to non-premixed modelling where the SGS variance is often approximated by algebraic closure [37,64], here it is obtained using its transport equation [23], as previously done in [40],

$$
\begin{aligned}
& \frac{\partial \bar{\rho} \widetilde{c^{\prime \prime 2}}}{\partial t}+\nabla \cdot\left(\bar{\rho} \tilde{\mathbf{u}} \widetilde{c^{\prime \prime 2}}\right) \approx \nabla \cdot\left[\left(\overline{\rho D}+\bar{\rho} \frac{v_{t}}{S c_{t}}\right) \nabla \widetilde{c^{\prime \prime 2}}\right] \\
& +2 \bar{\rho} \frac{v_{t}}{S c_{t}}(\nabla \tilde{c} \cdot \nabla \tilde{c})+2 \bar{\rho}\left(\widetilde{\dot{w}_{c} c}-\widetilde{\dot{w}_{c}} \tilde{c}\right)-2 \bar{\rho} \widetilde{\varepsilon_{c}}
\end{aligned}
$$


because of its close association with the SGS combustion, turbulence and molecular diffusion processes. Algebraic models [23] based on linear relaxation hypothesis using SGS turbulence time scale can not be used for premixed combustion [31] and thus the algebraic closure for SGS scalar dissipation rate in [77] is used:

$$
\widetilde{\varepsilon_{c}}=\Gamma\left[2 K_{c}^{*} \frac{S_{L}}{\delta_{t h}}+\left(C_{3}-\tau C_{4} D a_{S G S}\right)\left(\frac{2 u_{S G S}^{\prime}}{3 \Delta}\right)\right] \frac{\widetilde{c^{\prime \prime 2}}}{\beta_{c}}
$$

where $=1 \exp \left({ }_{5}{ }^{+}\right)$represents the normalized filter width with ${ }_{5}=0.75,{ }^{+}=/_{\text {th }}$ and being the LES filter width. The unstrained planar laminar flame speed $S_{L}$ and thermal thickness ${ }_{t}$ are calculated a priori using Chemkin PREMIX [68]. The SGS velocity is $u_{S G S}=\left(2 / 3 k_{S G S}\right)^{0.5}$ where $k_{S G S}$ is the SGS turbulent kinetic energy. The local SGS Damköhler and Karlowitz numbers are defined as $D a_{S G S}=\left(+/{u_{S G S}^{\prime}}^{+}\right)$and $K a_{S G S}=\left(\left(u_{S G S}^{\prime}\right)^{3} /{ }^{+}\right)^{0.5}$ where $u_{S G S}{ }^{+}=u_{S G S} / s_{L}$. The remaining model parameters are $K_{c}^{*}=0.79, C_{3}=1.5 \times K a_{S G S}{ }^{0.5}\left(1+K a_{S G S}{ }^{0.5}\right)^{1}$ and $C_{4}=1.1 \times\left(1+K a_{S G S}\right)^{0.4}$ where the normalized heat release is $=\left(\begin{array}{ll}T_{b} & T_{u}\end{array}\right) / T_{u}$. The parameter ${ }_{c}$ is set to 2.4 following the analysis conducted in [77]; a dynamic evaluation is not attempted here due to the limited influence reported in [72]. Equation (4) includes the effects of dilatation and strain rate as well as the contributions of chemical reaction and molecular dissipation to the scalar dissipation transport [77]. It is crucial to mention that these parameters are not arbitrary but derived from thermo-physical processes in hydrocarbon/air flames, meaning that the formulated CMC model lacks any tuning constant. The presented model has been successfully validated in [40] by means of measurements of $[78,79]$ for lean bluff body $\mathrm{CH}_{4}$-air flames at conditions far from and close to blow-off, respectively.

\subsection{Differential diffusion formulation}

In this section, a simplified extension of spatially-homogeneous CMC that accounts for differential diffusion is presented. The physical space species transport equation assuming non-unity constant Lewis numbers $L e_{i}$ is recalled here for completeness as

$$
\rho \frac{\partial Y_{i}}{\partial t}+\rho \mathbf{u} \cdot \nabla Y_{i}=\nabla \cdot\left(\rho \frac{\alpha}{L e_{i}} \nabla Y_{i}\right)+\dot{w}_{i}
$$

where represents the thermal diffusivity and $\dot{w}_{i}$ the instantaneous reaction rate of the $i$-species. Note that the correction velocity term accounting for gradients in the mixture molecular weight has not been included in the present 
formulation due to its negligible contribution, especially for lean mixtures [60]. Using Eq. (5) together with the instantaneous progress variable transport equation

$$
\rho \frac{\partial c}{\partial t}+\rho \mathbf{u} \cdot \nabla c=\nabla \cdot\left(\rho D_{c} \nabla c\right)+\dot{w}_{c}
$$

and exploiting the differential operators, the spatially-homogenous CMC governing equation is recovered in its conservative form as

$$
\frac{\partial Q_{i}}{\partial t}=\frac{1}{L e_{i}} \widetilde{N \mid \zeta} \frac{\partial^{2} Q_{i}}{\partial \zeta^{2}}+\widetilde{\dot{w}_{i} \mid \zeta}-\widetilde{\dot{w}_{c} \mid \zeta} \frac{\partial Q_{i}}{\partial \zeta}+\frac{D_{\zeta}}{\bar{\rho}}\left(\frac{1}{L e_{i}}-\frac{1}{L e_{c}}\right) \frac{\partial Q_{i}}{\partial \zeta}
$$

where the term $D=\nabla \cdot\left(D_{c} \nabla c\right)$ represents the conditional diffusion of $c$. Note that in progress variable space, differential diffusion is accounted for with a convective term, so that $D /^{-}\left(1 / L e_{i} \quad 1 / L e_{c}\right)$ is a convective velocity.

Assuming $D_{c}$ to be constant, the conditional diffusion of $c$ may be split into one term accounting for diffusion normal to the progress variable iso-surface and the other that is proportional to curvature, as previously done for both premixed [80] and non-premixed [56,81,82] flames, as

$$
\begin{array}{rlrl}
D & =\nabla \cdot\left(D_{c} \nabla_{c}\right) & \\
& =\mathbf{n} \cdot \nabla\left(D_{c} \mathbf{n} \cdot \nabla c\right) & & D_{c}|\nabla c| \nabla \cdot \mathbf{n} \\
& =\mathbf{n} \cdot \nabla\left(D_{c}|\nabla c|\right) & & D_{c}|\nabla c| \nabla \cdot \mathbf{n}
\end{array}
$$

with $\mathbf{n}=\nabla c /|\nabla c|$ defining the normal vector of the progress variable iso-surface, pointing towards the unburnt mixture.

The first term on the right hand side of Eq. (8) can be elaborated using the physical space derivative

$$
\nabla=\frac{\partial}{\partial c} \nabla c
$$

and the instantaneous scalar dissipation rate $N=D_{c}|\nabla c||\nabla c|$ resulting in

$$
\begin{aligned}
\mathbf{n} \cdot \nabla\left(D_{c}|\nabla c|\right) & =|\nabla c| \frac{\partial}{\partial c}\left(D_{c}|\nabla c|\right) \\
& =\left(\frac{N}{D_{c}}\right)^{1 / 2} \frac{\partial}{\partial c}\left[\left(D_{c}\right)^{1 / 2}(N)^{1 / 2}\right] \\
& =\frac{1}{2}\left[\frac{\partial}{\partial c}(N)+\frac{N}{D_{c}} \frac{\partial}{\partial c}\left(D_{c}\right)\right]
\end{aligned}
$$


In addition, defining the local curvature as $\quad=\nabla \cdot \mathbf{n}$, the second term of Eq. (8) reduces to

$$
D_{c}|\nabla c| \nabla \cdot \mathbf{n}=\quad\left(D_{c} N\right)^{1 / 2}
$$

Finally, the conditional diffusion of $c$ can be modelled as

$$
D=\frac{1}{2}\left[\frac{\partial}{\partial c}(N)+\frac{N}{D_{c}} \frac{\partial}{\partial c}\left(D_{c}\right)\right] \quad\left(D_{c} N\right)^{1 / 2}
$$

In the limit of unity Lewis number, the differential diffusion convective term of Eq. (7) disappears and the unity Lewis number formulation (Eq. (2)) is recovered. Consequently, any curvature effect vanishes as well.

The proposed methodology derives from flamelet theory [56] and does not involve any derivative of the PDF that gives rise to numerical instabilities. While it is less rigorous than other approaches where the conditional diffusion of the progress variable is directly linked to the PDF first derivative [35], it represents a robust model able to provide a reasonable approximation of the involved processes, as shown in the next section.

\section{Results}

\subsection{Spatially-homogeneous planar CMC}

The effect of differential diffusion on the flame structure is evaluated and discussed in the following for both laminar and turbulent-like (as it will be addressed later) cases. In this regard, spatially-homogenous laminar unstretched (curvature is expressly set to zero) CMC simulations are conducted for both unity Lewis number and full transport formulations and compared with calculations performed using the PREMIX library [68] from the CHEMKIN package [83]. The latter solves the steady-state mass, species and energy conservation equations for a freely propagating adiabatic planar flame using a Newton-iteration technique. Sufficient grid points (in the order of 1500) were employed to ensure a converged solution and the methane oxidation chemistry is described by the GRI-Mech 3.0 [84] mechanism.

The premixed $\mathrm{CH}_{4}$-air mixture is considered at ambient conditions ( $T_{u}=298 \mathrm{~K}$ and $p=1 \mathrm{bar}$ ) and has the same thermochemical properties of the flame A4 investigated experimentally in [78]: the equivalence ratio is $=0.64$ resulting in an unstretched laminar flame speed of $s_{L}=0.14 \mathrm{~m} / \mathrm{s}$ and an unstretched laminar flame thickness of ${ }_{t h}=0.81 \mathrm{~mm}$. In Fig. 1, blue lines represent the unity Lewis number solution for both CMC (dashed) and PREMIX (solid) for the most important species of the adopted chemical mechanism. For the former, the Lewis number of each species has been set to 1 , so that the additional terms related to differential diffusion of the CMC transport equation are 
dropped; for the latter, the molecular diffusivity of each species has been imposed equal to the mixture thermal diffusivity. One can observe that CMC recovers very well the PREMIX solution for the species mass fractions shown.
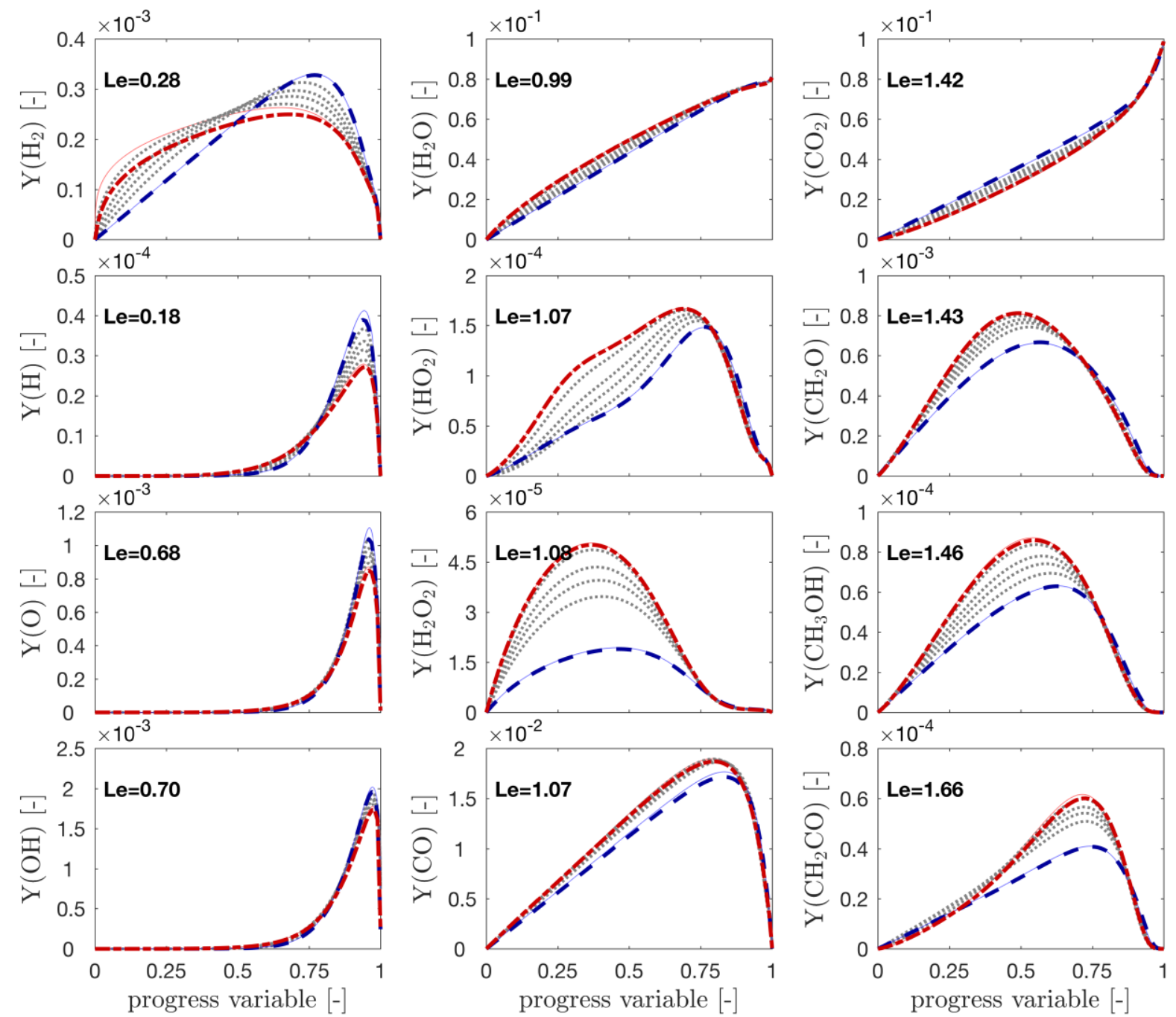

Fig. 1. Comparison between unity Lewis number (PREMIX: solid blue line, CMC: dashed blue line) and full transport (PREMIX: solid red line, CMC: dash-dot red line) unstretched calculations for different species mass fractions. The 4 dotted black lines represent PREMIX full transport calculations using a supplementary turbulent diffusivity $D_{\text {turb }}=\{50,200,400,1000\} \quad 10{ }^{6} \mathbf{m}^{2} / \mathbf{s}$

Furthermore, the red lines represent the full transport solution for both CMC (dash-dot) and PREMIX (solid). The Lewis numbers labelled in the graph and mentioned throughout this manuscript are indicatively expressed at the unburnt mixture condition, whereas they are accurately evaluated as a function of temperature and mixture composition during the computations. Overall, the flame structure differs significantly for the unity Lewis number solution, suggesting that for this specific flame differential diffusion effects cannot be neglected, although the laminar flame speeds between both transport formulations only vary by $2 \%$. The response of the CMC equations including differential diffusion agrees very well with the reference solution, which was obtained with a set of equations of different formalism and numerics. For 
species with low Lewis numbers (e.g. $\mathrm{H}_{2}$ ), preferential transport enhances diffusion from the higher towards the lower values of the progress variable. This is partly explained by the evolution of the conditional diffusion exhibited in Fig. 2 (right), showing an inflection point around $c=0.7$. For species with Lewis numbers well above unity (e.g. $\left.\mathrm{CH}_{2} \mathrm{CO}\right)$ a weak opposite trend can be observed. Note that all species tend to reach a new equilibrium when diffusing across the conditioning variable coordinate, so that chemical effects are influencing the final solution and cannot be decoupled.

The flame propagation in physical space in flamelet-based approaches is generally described by equations Eq. (1) and Eq. (3), therefore it is closely related to the progress variable reaction rate, rather than to species distributions. Fig. 2 (left) shows the progress variable reaction rate for the unity Lewis number solution (blue) and full transport calculation (red) for both CMC (dashed) and PREMIX (solid) calculations. The effect of the differential transport is significant and well captured by CMC: when the full transport is considered, the reaction rate becomes wider covering a larger portion of the flame preheat layer and its peak reduces.
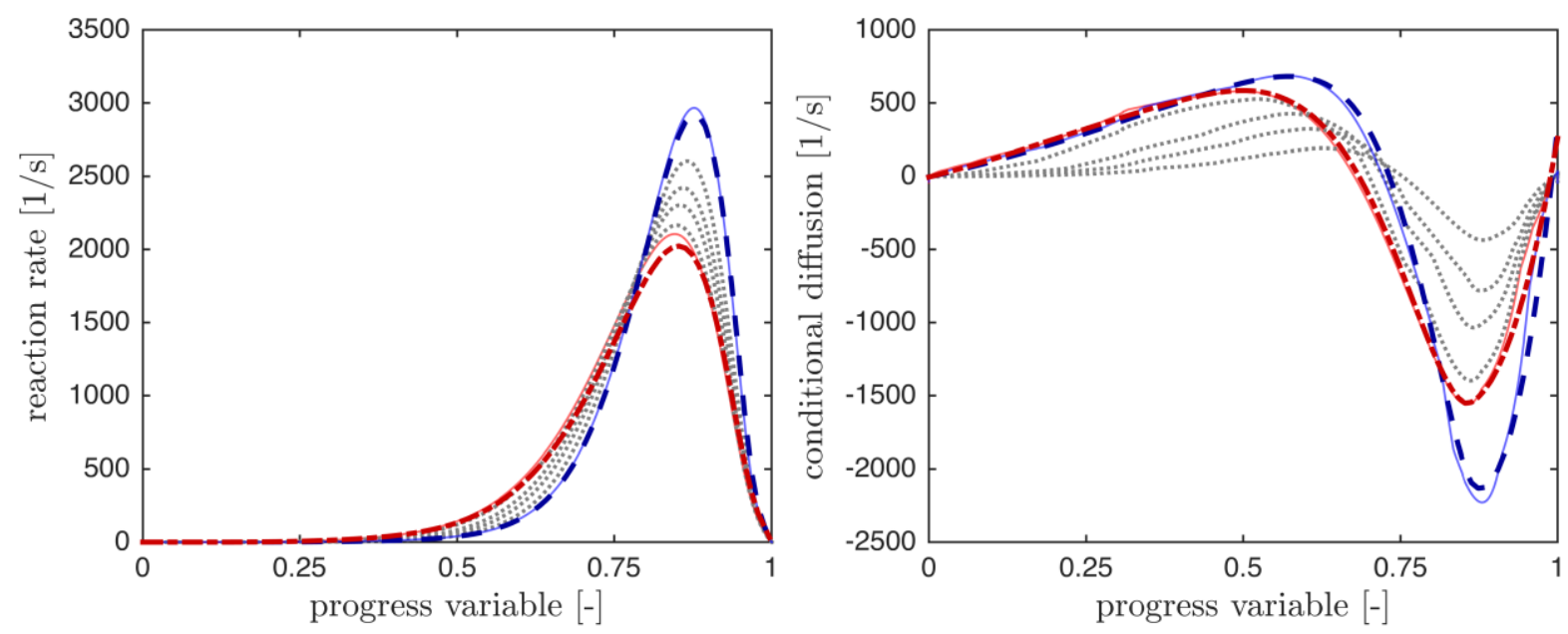

Fig. 2. Comparison between unity Lewis number (PREMIX: solid blue line, CMC: dashed blue line) and full transport (PREMIX: solid red line, CMC: dash-dot red line) unstretched calculations for reaction rate (left) and conditional diffusion of the progress variable (right). The four dotted black lines represent PREMIX full transport calculations using a supplementary turbulent diffusivity $D_{\text {turb }}=\{50,200,400,1000\} \quad 10^{6} \mathbf{m}^{2} / \mathbf{s}$

In order to assess the relative importance of the terms constituting the spatially-homogeneous CMC governing equation, a budget analysis is shown in Fig. 3 for three selected species $\left(\mathrm{CH}_{4}, \mathrm{CH}_{2} \mathrm{O}, \mathrm{H}_{2}\right)$ and temperature. For this purpose, Eq. (7) is recalled here for readability and split up in its source/sink terms as

$$
\underbrace{\frac{\partial Q_{i}}{\partial t}}_{\text {rate of change }}=\underbrace{\frac{1}{L e_{i}} \widetilde{N \mid \zeta} \frac{\partial^{2} Q_{i}}{\partial \zeta^{2}}}_{\text {molecular diffusion }}+\underbrace{\widetilde{\dot{w}_{i} \mid \zeta}}_{\text {reaction rate }}-\underbrace{\widetilde{\dot{w}_{c} \mid \zeta \frac{\partial Q_{i}}{\partial \zeta}}}_{\begin{array}{c}
\text { convection in } \\
\text { sample space }
\end{array}}+\underbrace{\frac{D_{\zeta}}{\rho \mid \zeta}\left(\frac{1}{L e_{i}}-\frac{1}{L e_{c}}\right) \frac{\partial Q_{i}}{\partial \zeta}}_{\text {differential diffusion }}
$$


where the unsteady term is observed to be zero for all variables due to steady convergence as observed in Fig. 3 (black line with cross markers), supporting the neglecting of the correction velocity term in Eq. (5).
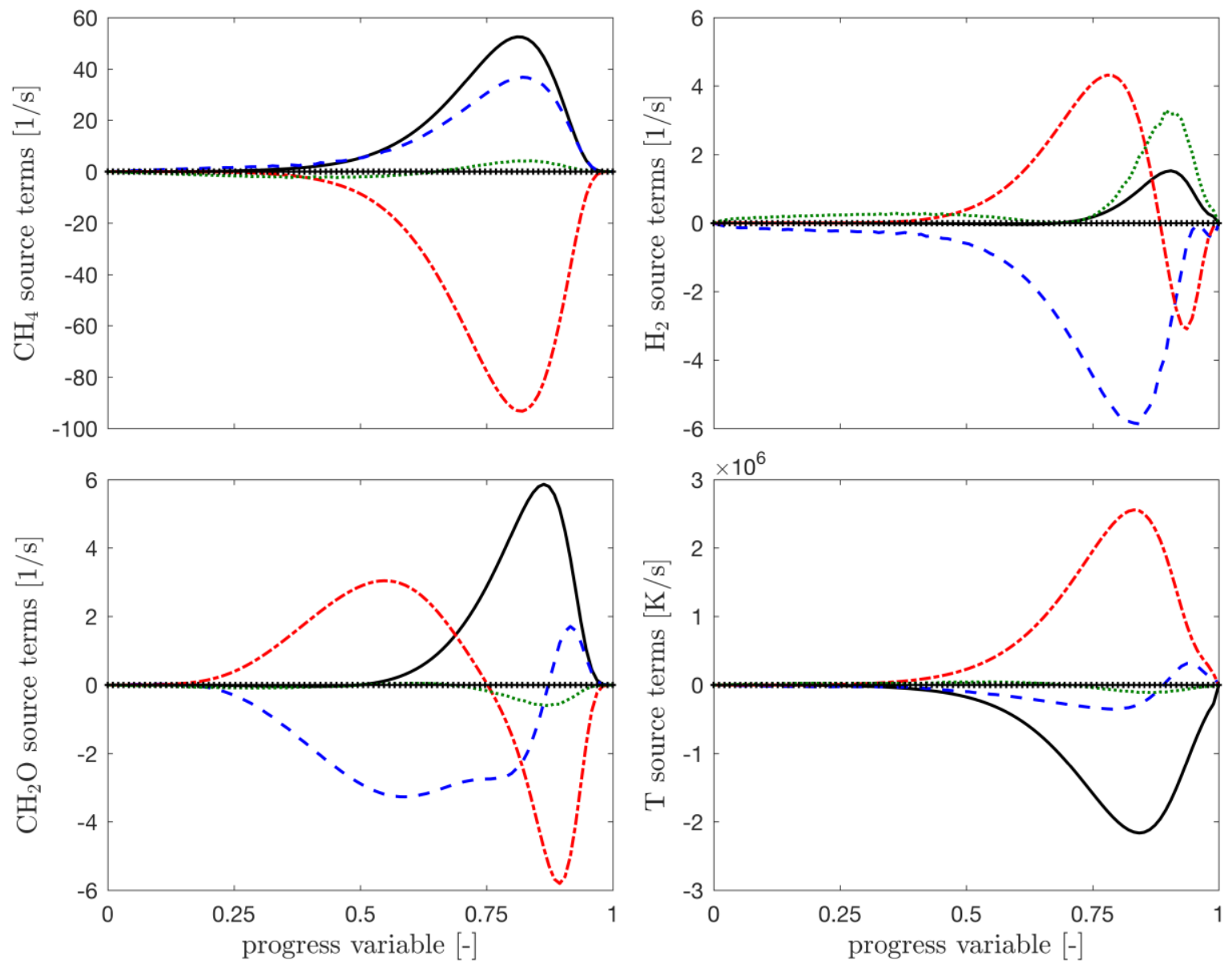

Fig. 3. Budget analysis of the unstretched CMC full transport equation for selected species and temperature: reaction rate (dash-dot red line), convection in sample space (solid black line), molecular diffusion (dashed blue line) and differential diffusion (dotted green line) contributions. The black line with cross markers represents the sum of all contributions.

The reaction rate (dash-dot red line) is expectedly a dominant term for all species and temperature. On the other hand, convection in sample space (solid black line) is proportional to the conditioning variable reaction rate (in this case oxygen) and the species first derivative, therefore remaining usually positive for species and non-negligible. The micromixing within the flame front is described by the molecular diffusion term (dashed blue line), proportional to the scalar dissipation rate, which is in the same order of magnitude as the chemical term. The differential diffusion term is proportional to the conditional diffusion of $c, D$, the species first derivative and an algebraic expression including the species Lewis number that determines its magnitude. As previously mentioned, when the species Lewis number tends to unity (as for $\mathrm{CH}_{4}$ ) this term becomes zero and the unity Lewis number CMC formulation is recovered. On the contrary, when the species Lewis number largely deviates from unity (as for $\mathrm{H}_{2}$ ), differential diffusion starts dominating the 
molecular transport. Therefore, it becomes apparent that for species with Lewis numbers far from unity an accurate description of the conditional diffusion plays a crucial role. The model expressed in Eq. (12) is therefore evaluated in Fig. 2 (right) and compared with the spatial fully-resolved PREMIX simulation where it has been calculated directly as $D=\nabla \cdot\left(D_{c} \nabla c\right)$. The excellent agreement between CMC and the reference solution supports the constant $D_{c}$ assumption adopted in deriving Eq. (8).

In order to mimic the turbulence effect on the flame structure and assuming that turbulence mixes all scalars by the same amount, the PREMIX code has been augmented by an additional turbulent diffusivity, $D_{\text {turb }}$, supplementing the molecular diffusivity of each species, $D_{i}$, following the same approach as [47]

$$
D_{e f f, i}=D_{i}+D_{t u r b}
$$

and accordingly

$$
\text { eff }=+c_{p} D_{\text {turb }}
$$

where $\quad$ and $c_{p}$ are the mixture conductivity, density and heat capacity, respectively. Consistent with premixed flame theory, as the turbulent diffusivity is increased the resulting flame speed and flame thickness rise proportional to $D_{e f f, i}{ }^{1 / 2}$, as shown in Fig. 4. It is worth mentioning that in this approach the turbulent diffusivity is assumed to remain constant through the flame front, whereas in reality it is expected to vary due to flow laminarisation. Nevertheless, this numerical experiment allows a preliminary insight into the importance of the differential diffusion effect on the flame structure at different prescribed turbulence intensities.

Fig. 1 shows four dotted black lines representing PREMIX full transport calculations for four turbulent diffusivities $D_{\text {turb }}=\{50,200,400,1000\} \quad 10^{6} \mathrm{~m}^{2} / \mathrm{s}$. At $D_{\text {turb }}=50 \mathrm{~m}^{2} / \mathrm{s}$ the flame structure recovers very well the laminar solution, due to the molecular diffusion dominating the transport process. Consistent with observations in $[47,52]$, with increasing turbulence the solution tends towards the unity Lewis number solution as the molecular diffusion becomes negligible and consequently differential diffusion effects weaken. In the CMC context, turbulence induces a flame broadening - as also observed in [7,85] - that reduces the conditional diffusion absolute value (as shown in Fig. 2, dotted lines) and accordingly the differential diffusion contribution. It is worth mentioning that the differential diffusion model contains the scalar dissipation rate, therefore turbulence effects described through the scalar dissipation rate are consistently considered in Eq. (12). 


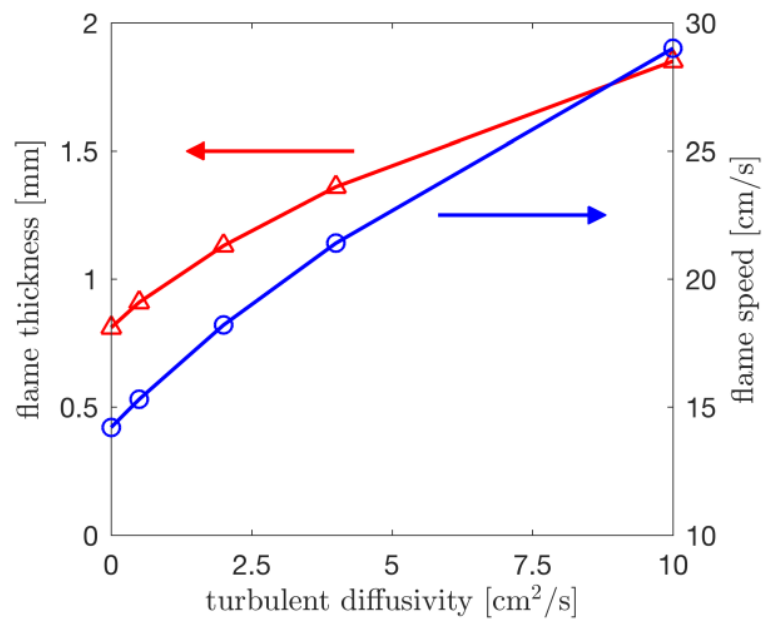

Fig. 4. Flame speed (blue circles) and flame thickness (red triangles) as a function of the turbulent diffusivity.

\subsection{Spatially-homogeneous CMC with curvature effects}

In the previous section, the CMC formulation has been carefully validated by means of fully-resolved planar unstretched simulations performed using the PREMIX library. In the following, the solution of curved laminar flames of different mixtures are computed with CMC and compared with the respective unstretched solution. It is worth to mention that only the full transport CMC formulation is suitable for this investigation, since curvature effects are only relevant in case preferential transport occurs. Moreover, neglected tangential diffusion effects may arise when the Lewis numbers are significant far from unity. This means that the sole conditioning direction orthogonal to the flame front may be insufficient to account for all transport phenomena and thereby additional curvilinear coordinates may be necessary, at the cost of undesired modelling uncertainties. Accordingly, while calculations conducted in this section could potentially slightly deviate from exact solutions, this does not undermine the scope of this analysis aiming at discussing the effective trends involved with preferential diffusion in the presence of flame curvature.

Two different mixtures are considered at ambient conditions: the same lean $\mathrm{CH}_{4}$-air mixture of the previous section and an $\mathrm{H}_{2}$-air mixture (with $=0.4, s_{L}=0.22 \mathrm{~m} / \mathrm{s}$ and ${ }_{t h}=0.63 \mathrm{~mm}$ ). The lean $\mathrm{H}_{2}$-air mixture has been considered here due to the similar response to stretch as the $\mathrm{CH}_{4}$-air flame compared to heavier hydrocarbon mixtures [86]. 

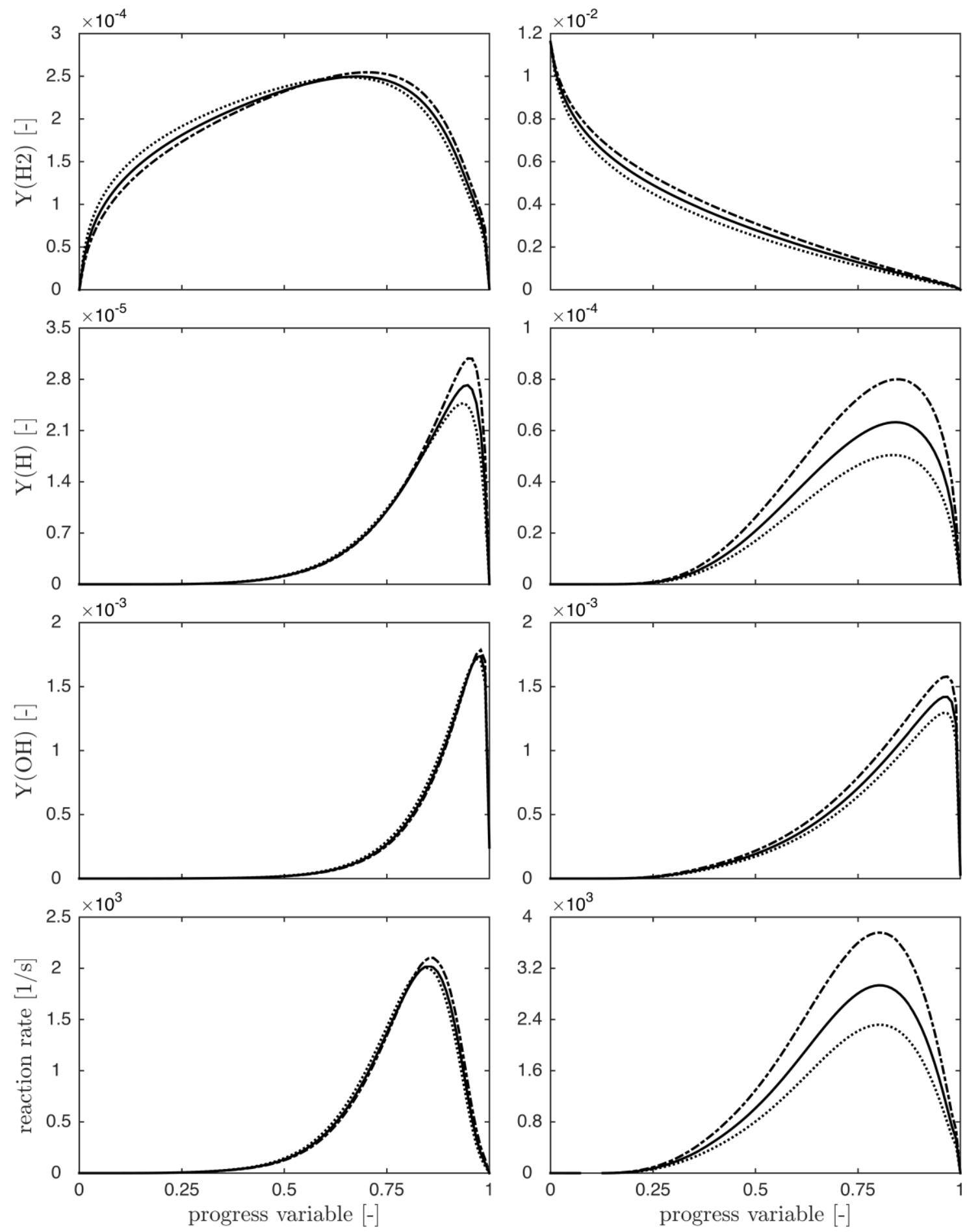

Fig. 5. Influence of flame curvature on species mass fractions and reaction rate for a $\mathrm{CH}_{4}$-air flame with $=0.64$ (left) and a $\mathbf{H}_{2}$-air flame with $=0.4$ (right): $\mathbf{C M C}$ full transport calculations with constant curvatures $=\{0,+400,400\} \mathbf{1} / \mathbf{m}$ represented by solid line, dash-dot line and dotted line, respectively.

Fig. 5 shows the flame structure in terms of selected species mass fractions and progress variable reaction rate for the $\mathrm{CH}_{4}$-air (left) and the $\mathrm{H}_{2}$-air (right) flames. The unstretched - here means $=0$ due to the absence of hydrodynamic 
strain - reference solution (solid line) is compared with computations considering a constant positive curvature of $=+4001 / \mathrm{m}$ (dash-dot line) and a negative one of $=4001 / \mathrm{m}$ (dotted line), representing the maximum measured values in [78]. For the $\mathrm{CH}_{4}$-air case, curvature is observed to noticeably affect $\mathrm{H}_{2}(L e \quad 0.28)$ and $\mathrm{H}$ ( Le 0.18 ) distributions, whereas an influence can hardly be observed for $\mathrm{OH}$ ( $L e \quad 0.70$ ) due to its Lewis number closer to unity. Despite different species deviate from the unstretched solution, the progress variable conditional reaction rate is observed to remain almost unaltered.

A different behaviour is observed for the lean $\mathrm{H}_{2}$-air flame, where curvature evidently affects all the selected species of Fig. 5 and thereby the resulting progress variable conditional reaction rate. The remarkably different response of the flames to curvature can be explained exploiting the asymptotic theory of premixed flames, where the flame effective Lewis number can be expressed as a weighted average of the Lewis numbers of the two reactants as [51]

$$
L e_{e f f}=\left\{\begin{array}{lll}
\frac{L e_{O}+\mathcal{A} \cdot L e_{F}}{1+\mathcal{A}}, & \text { if } & <1 \\
\frac{L e_{F}+\mathcal{A} \cdot L e_{O}}{1+\mathcal{A}}, & \text { if } & >1
\end{array}\right.
$$

where $L e_{F}$ and $L e_{O}$ are the Lewis numbers of fuel and oxygen, respectively, and

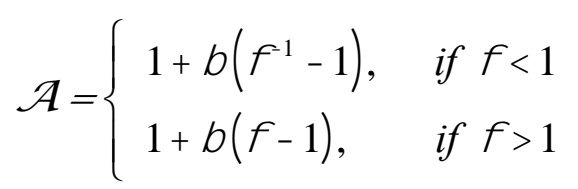

Assuming a Zel'dovich number of about 6 for $\mathrm{CH}_{4}$-air [87] and $\mathrm{H}_{2}$-air, the effective Lewis numbers are estimated as 0.99 and 0.33 , respectively, implying that the $\mathrm{H}_{2}$-air flame is prone to show a burning velocity (and therefore progress variable reaction rate) dependence on flame curvature, as also confirmed in [61]. These findings are also consistent with [87] where for a $\mathrm{CH}_{4}$-air mixture of comparable thermo-physical properties, a relatively small influence of flame stretch on the local burning rate is reported.

Summarising, the effect of flame stretch on the conditional progress variable reaction rate, and thereby on the resulting flame propagation, is evaluated for two flames of significantly different effective Lewis numbers: the investigated cases suggest that the term of Eq. (12) proportional to curvature can be neglected in the case of mixtures with effective Lewis numbers close to unity. 


\subsection{LES-CMC of a bluff body stabilised flame}

In the previous section, the differential diffusion formulation for $\mathrm{CMC}$ has been quantitatively validated by means of well-resolved PREMIX simulations for laminar flames. Moreover, an analysis mimicking the turbulence effect on the flame structure suggested that at high turbulent Reynolds numbers turbulent transport overcomes the molecular one, so that differential diffusion may become negligible. The validity of this hypothesis will be assessed in the following, where 3-dimensional LES-CMC simulations are conducted for both the non-unity and the unity Lewis number formulations and qualitatively compared with experimental data. Consistent with previous findings, curvature effects are neglected.

\subsubsection{Problem setup and numerical details}

The flame considered is the lean turbulent premixed bluff body flame investigated experimentally in [78,79] and numerically in [40]. The burner is open to the environment and consists of a conical $45^{\circ}$ half-angle bluff body with a diameter of $25 \mathrm{~mm}$, concentrically mounted within the central inlet pipe with a diameter of $35 \mathrm{~mm}$, carrying the premixed $\mathrm{CH}_{4}$-air charge at ambient conditions. The reported bulk velocity measured at the burner exit at unignited condition is $21.6 \mathrm{~m} / \mathrm{s}$, with $3 \%$ uncertainty. The considered flame A4 has the same thermo-physical properties as the one investigated in the previous section, here repeated for readability: $=0.64, s_{L}=0.14 \mathrm{~m} / \mathrm{s}$ and ${ }_{t h}=0.81 \mathrm{~mm}$.

The LES computational domain is shown in Fig. 6 and consists of a cylindrical shape with a diameter of $140 \mathrm{~mm}$ and a length of $250 \mathrm{~mm}$ comprising a total of 1.8 million hexahedral cells aligned in an $O$-ring topology. Note that the number of elements has been decreased by a factor of 2 compared to the previous work [40], for reasons that will be discussed in the next section. The minimum grid size is $0.6 \mathrm{~mm}$ and includes most of the region above the bluff body, where the flame is expected; bias functions are used to ensure a smooth grid increase towards the boundaries. The base side of the domain is set as inlet with a top-hat velocity profile and zero-gradient pressure for both premixed charge and air co-flow. No synthetic turbulence treatment is adopted since all the turbulent kinetic energy is expected to stem from the shear layer. Non-reflecting pressure (atmospheric) outlets are prescribed elsewhere and zero-gradients for other variables. The finite volume CMC formulation exploits the weaker dependence of conditional averages compared to unconditional counterparts by employing a coarser, unstructured CMC mesh, comprising 1600 polyhedral elements concentrated in the flame region above the bluff body $(50 \times 50 \times 80 \mathrm{~mm})$. This refinement is expected to capture the influence of the local scalar dissipation rate on the conditional averages. Progress variable sample space is discretized with 96 uniformly distributed points. Clustering of points in the vicinity of the burnt state as employed in [40] was found to be unnecessary, due to the relatively smooth profiles in conditional space. Using an equidistant grid in sample space further avoids numerical instability when differential diffusion is considered. 


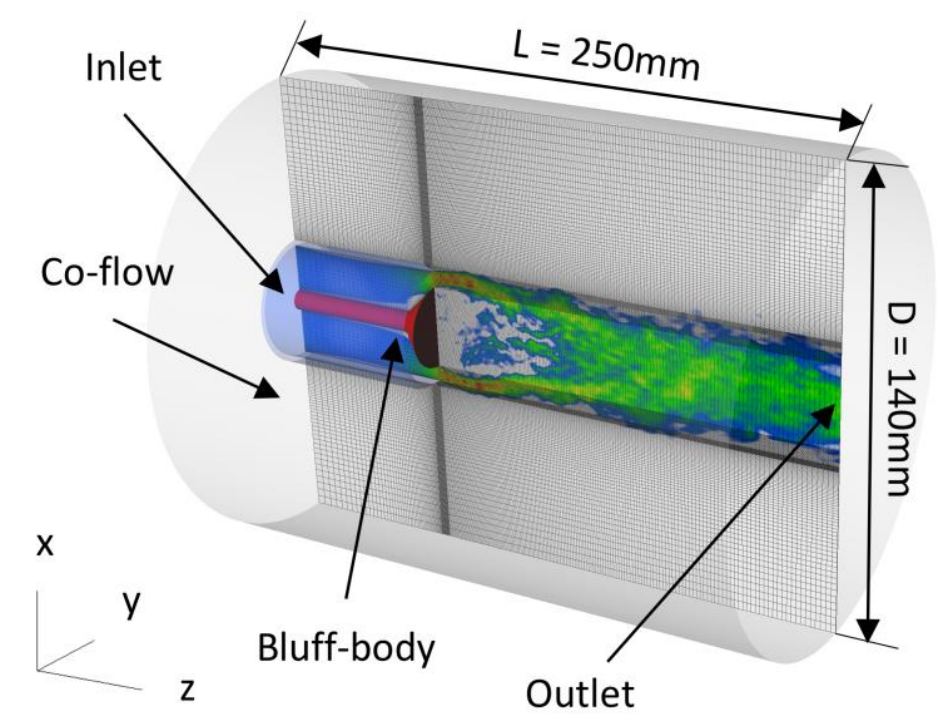

Fig. 6. Schematic of the computational grid with superimposed instantaneous velocity field and bluff body solid (red region).

OpenFOAM v3.0 is adopted to solve the LES equations, which are implicitly filtered using the filter width $=V_{L E S}^{1 / 3}$, where $V_{L E S}$ is the cell volume. Velocity and pressure fields are solved with the 'PIMPLE' pressure-correction algorithm in a compressible formulation using the in-built OpenFOAM solver, where a transient backward second-order implicit time scheme is employed. Differently from [40], in this study the more advanced one equation eddy-viscosity model with default parameters is adopted. The OpenFOAM solver has been extended to solve additional equations for the mean progress variable, Eq. (1), and its variance, Eq. (3), allowing the evaluation of the FDF functions at LES resolution. The flow field solver time step is set to $1 \mu$ s ensuring a maximal Courant number of 0.3 throughout the entire domain at all time steps. The implementation of an internal CMC sub-step (here of $0.2 \mu$ s) was additionally necessary to handle the more restrictive time step constraint dictated by the differential diffusion formulation, without excessively compromising computational efficiency.

The CMC solver is initialized with the conditional averages calculated a priori using PREMIX [68], representing a laminar freely propagating flame. A full operator-splitting technique is adopted to separately integrate physical space transport, molecular mixing, and reactive terms. For the latter, a load balance approach based on progress variable is implemented to further reduce computational effort [64]. A first order explicit Euler time scheme is employed in CMC to integrate convection and turbulent flux, while the more robust VODPK solver with stiff settings (internal sub-step, up to third order accuracy) [88] is adopted to treat diffusion in progress variable space and chemistry. The GRI-3 reduced version mechanism developed in [89] is employed, consisting in 19 species and 15 lumped steps. The interfacing of LESCMC occurs at each iteration so that OpenFOAM provides $\tilde{\mathbf{u}}, \tilde{\boldsymbol{c}}, \widetilde{c^{\prime \prime 2}}, \tilde{p}$ and $\widetilde{N_{c}}$ and after the CMC step the updated 
species mass fractions $\tilde{Y}_{i, C M C}=\int_{0}^{1} Q_{i} \tilde{p}(\zeta) \partial \zeta$ are returned to the flow field solver. Under the assumption that the flame brush never interacts with the entrainment air, the effective species mass fractions are corrected as $\tilde{Y}_{i}=\tilde{z} \cdot \tilde{Y}_{i, C M C}+(1-\tilde{z}) \cdot Y_{i, a i r}$, where $Y_{i, \text { air }}$ represents the boundary condition of the $i$-species at the air co-flow inlet.

The mixing state is computed with an additional transport equation, where $\tilde{z}=1$ represents the prescribed composition of the inlet jet and $\tilde{z}=0$ indicates the ambient air. Filtered temperature is estimated at the CFD resolution after solving the chemico-thermal enthalpy conservation equation.

\subsubsection{Cold flow}

In this study, a sensitivity analysis on the non-reacting flow has been conducted with the scope of identifying the coarsest possible grid able to capture the complex flow field above the bluff body, not only to reduce computational effort, but rather to enhance as much as possible the impact of the combustion model on the final solution.
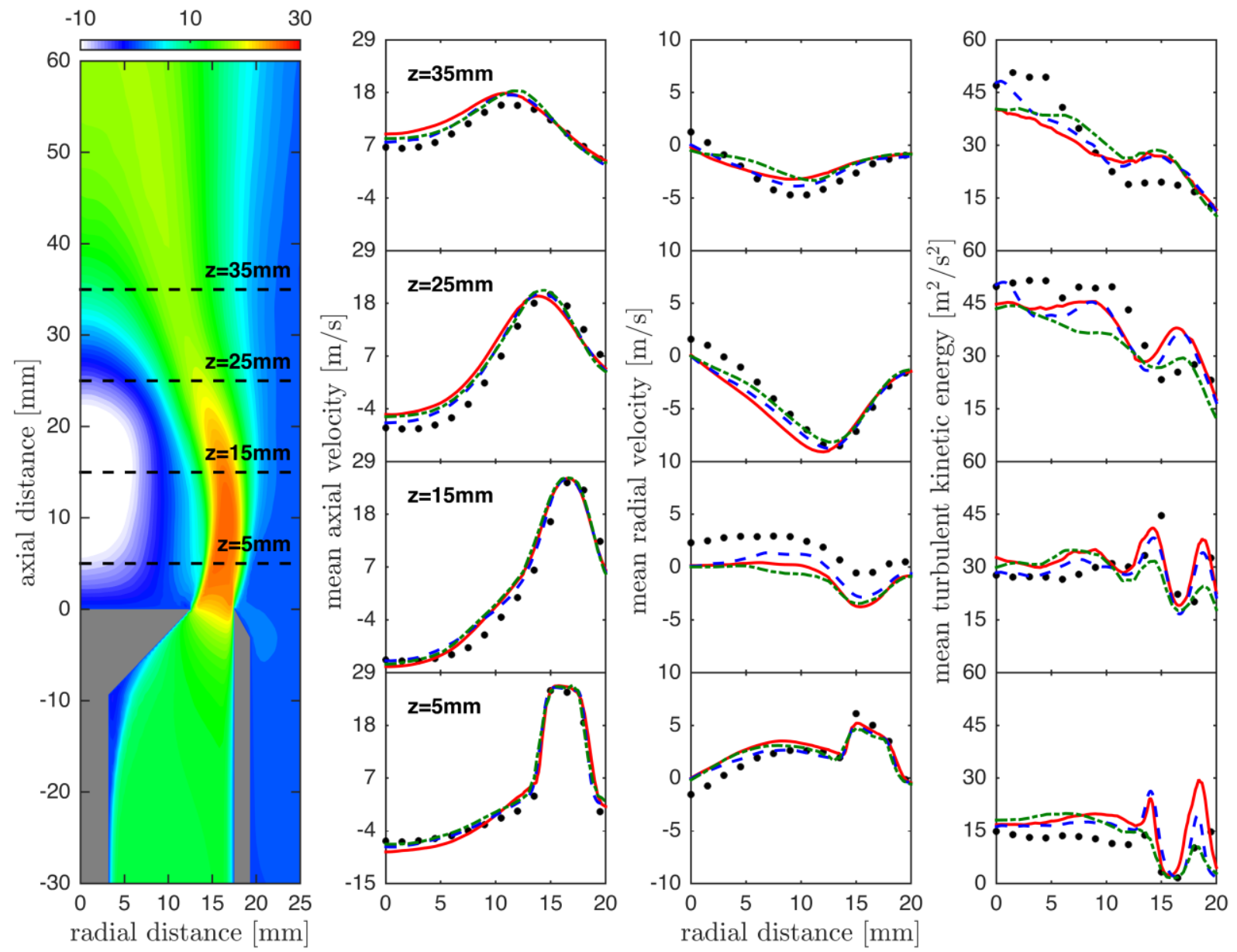

Fig. 7. Comparison between experiment [78] (circles) and simulations (lines) for mean axial and radial velocities and turbulent kinetic energy at four axial distances $\mathrm{z}=5,15,25$ and $35 \mathrm{~mm}$. Computational grids with $0.9,1.8$ and 3.6 million of elements are 
represented by dash-dot green, solid red and dashed blue lines, respectively. The computed mean axial velocity field is shown on the left together with the bluff body solid (grey region).

Mean axial (left) and radial (middle) velocity as well as mean turbulent kinetic energy (right) are quantitatively compared with experimental data in Fig. 7 for a 0.9 (dash-dot green line), 1.8 (solid red line) and 3.6 (dashed blue line) million of elements grid. The computed instantaneous quantities have been time-averaged at time-stepping frequency over 6 flowthrough times starting from a fully developed solution to ensure statistically converged results, following the same procedure adopted in [40]. All the considered computational grids capture the mean axial and radial velocities reasonably well, without manifesting any remarkable improvement with respect to the grid size. The larger discrepancies observed in both mean radial velocity and turbulent kinetic energy are attributed to the turbulence model, as also discussed in [90]. A noticeable improvement is observed for the computed mean turbulent kinetic energy as the number of computational elements is increased. Overall, the 1.8 and 3.6 million of elements grids exhibit comparable results, whereas the 0.9 million of elements grid is not capable of reproducing the fluctuations in the mean turbulent kinetic energy along the radial axis, especially in the downstream region. For this reason, the 1.8 million of elements grid is employed for the reactive simulation: the respective average cell size in the flame region $(0.6 \mathrm{~mm})$ is therefore comparable to the laminar flame thickness $(0.81 \mathrm{~mm})$.

\subsubsection{Reactive flow}

The LES-CMC numerical framework used in this study has been comprehensively validated for flame A4 in a previous study [40] using experimental data of [78,79] in terms of qualitative ( $\mathrm{OH}$ and mean progress variable fields) and quantitative (flame surface density and local flame curvature) comparisons. Here, only the qualitative influence of differential diffusion on the mean flame morphology in case of significant turbulence-chemistry interaction is discussed. 


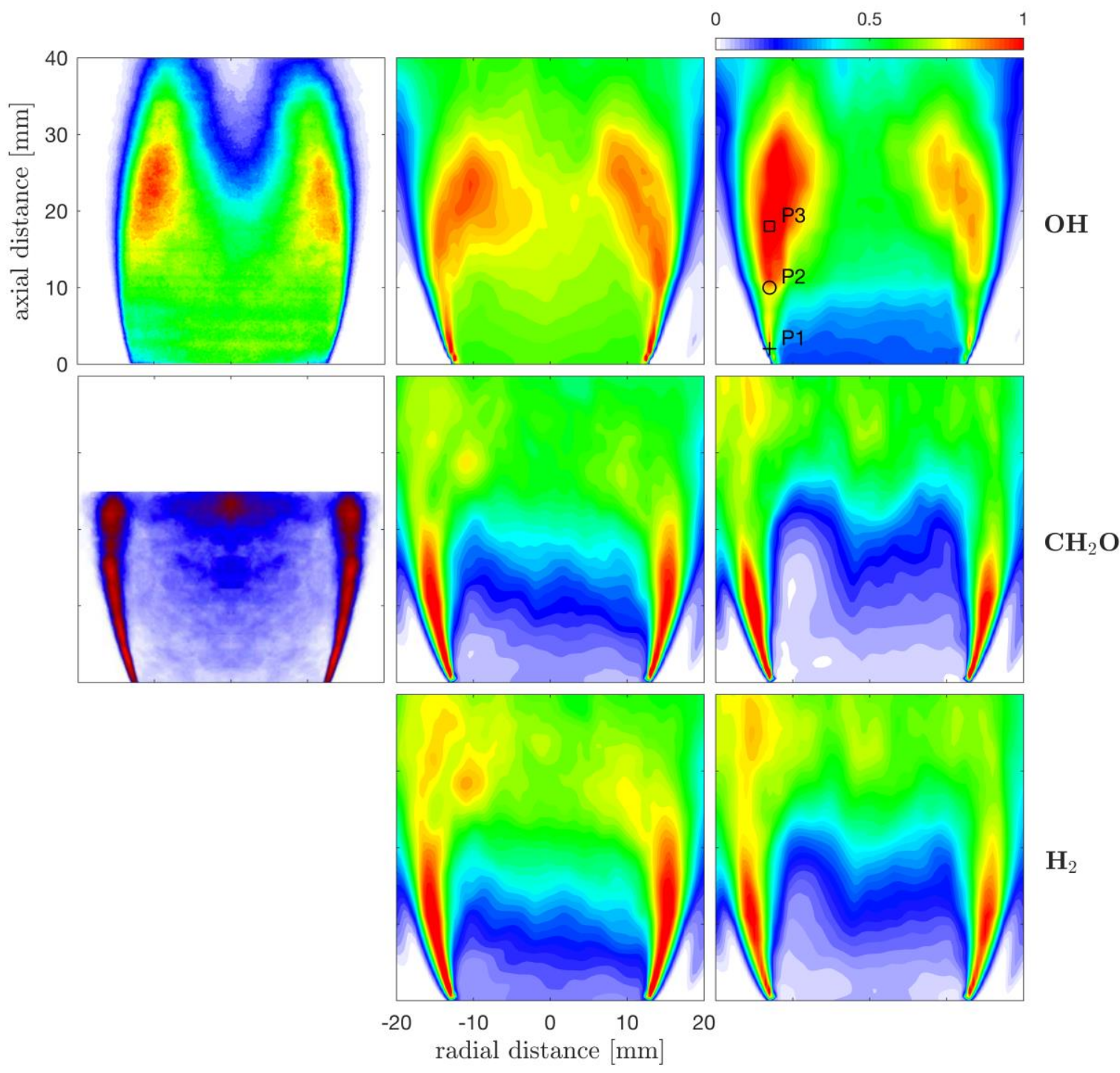

Fig. 8. Qualitative comparison of mean $\mathrm{OH}$ (top), $\mathrm{CH}_{2} \mathrm{O}$ (middle) and $\mathrm{H}_{2}$ (bottom) distributions between experiment (left) and simulations (unity Lewis number: center, full transport: right) for flame A4 in arbitrary units. Experimental data for OH and $\mathrm{CH}_{2} \mathrm{O}$ are taken from [78] and [79], respectively; no data for $\mathbf{H}_{2}$ are available. Points P1 (cross), P2 (circle) and P3 (square) indicate three different positions inside the flame brush.

Fig. 8 shows the qualitative comparison of mean $\mathrm{OH}$ (top), $\mathrm{CH}_{2} \mathrm{O}$ (middle) and $\mathrm{H}_{2}$ (bottom) distributions between experiment [78,79] (left) and simulations for the unity Lewis number (center) and full transport (right) formulations. Note that the experimental OH-PLIF data shown in the top row of Fig. 8 is the correct average; Fig. 6 in reference [78] unfortunately has a mistake (Dr. Kariuki, private communication, Nov. 2015). The numerical configuration (schemes, time-step, averaging procedure, etc.) between both simulations is identical in order to avoid undesired side effects. Overall, both computations reproduce the M-shaped flame observed experimentally caused by the OH penetrating the recirculation zone, as discussed in detail in [40]. The major differences between the two computations are the slight 
flame height under prediction by the unity Lewis number formulation and the underestimation of the $\mathrm{OH}$ mass fraction inside the recirculation zone by the full transport CMC. Some differences in the $\mathrm{H}_{2}$ distribution inside the recirculation zone are also evident. Nevertheless, the mean $\mathrm{OH}, \mathrm{H}_{2}$ and $\mathrm{CH}_{2} \mathrm{O}$ distributions between both simulations is qualitatively comparable, suggesting that to a certain extent for large Karlovitz numbers (here from 1 to 10 [78]) the influence of differential diffusion is not substantial.

As earlier anticipated, the reason of this behaviour is attributed to turbulence: in the conventional physical space transport, the turbulent diffusivity dominates the molecular one, thereby reducing the budget of the differential diffusion contribution, as discussed in the context of Fig. 1 and Fig. 2. On the other hand, the same effect is obtained in CMC with turbulence causing a reduction of the conditional scalar dissipation rate (CSDR).
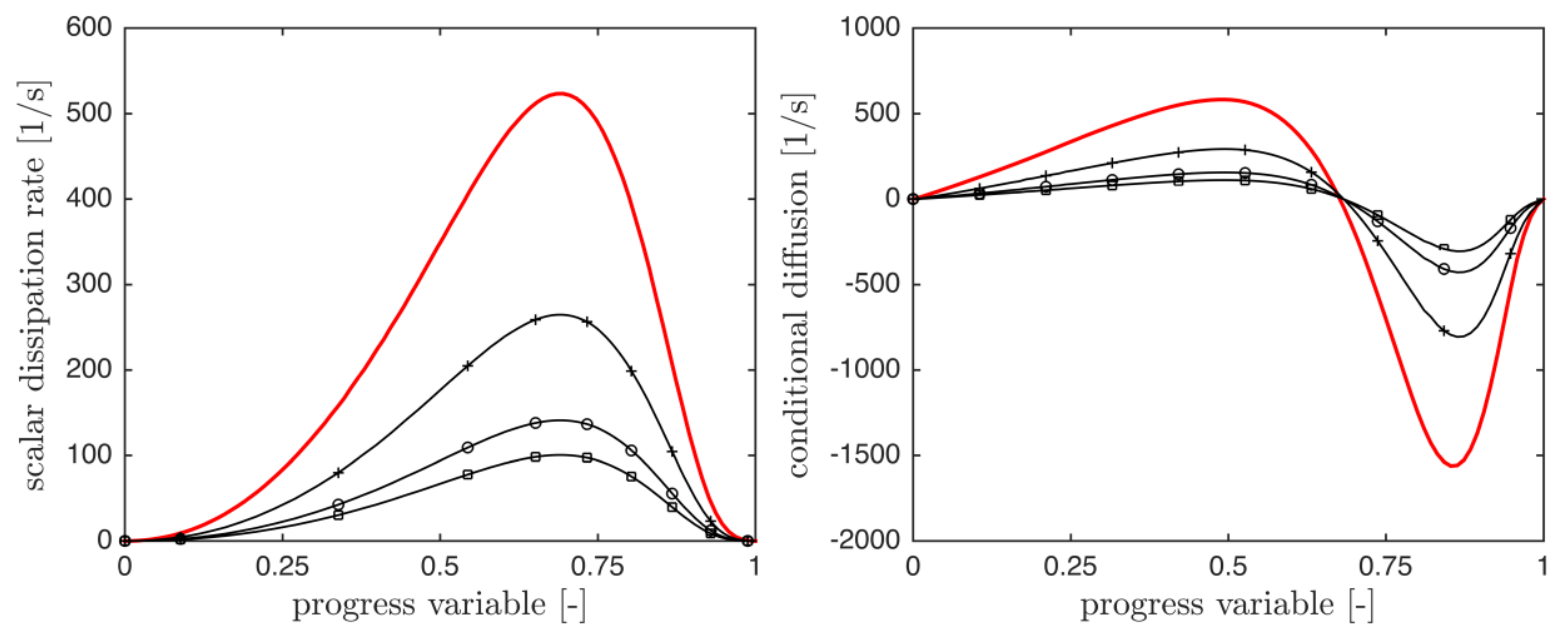

Fig. 9. Scalar dissipation rate (left) and conditional diffusion of $c$ (right) for the laminar solution (solid red line) and the LESCMC calculation at 3 different positions P1 (cross markers), P2 (circle markers) and P3 (square markers) inside the flame brush.

Fig. 9 shows the CSDR for the laminar solution (solid red line) and the LES-CMC computation with differential diffusion at three different locations inside the flame brush (P1: cross markers, P2: circle markers, P3: square markers). As the CSDR decreases, the respective conditional diffusion of $c(C D)$ also reduces, diminishing the importance of preferential transport. This is fully consistent to what observed in Fig. 2 when an artificial turbulent diffusivity was superimposed to the laminar one. Interestingly, at the anchoring location P1 where the flame brush is still relatively thin, both the CSDR and the CD are closer to the laminar solution so that the differential diffusion contribution becomes more important. Differently, at locations in the flame brush P2 and P3, turbulence broadens the flame front decreasing the CSDR and accordingly the importance of preferential transport. 


\section{Conclusions}

The finite volume LES-CMC numerical framework for premixed combustion developed in a previous study [Farrace et al., Proc. Combust. Inst. 36 (2017)] has been extended to account for differential diffusion. The non-unity Lewis number CMC transport equation shows an additional convective term in sample space proportional to the conditional diffusion of the progress variable, that in turn accounts for diffusion normal to the flame front and curvature-induced effects. Planar laminar simulations are first performed using a spatially-homogeneous non-unity Lewis number CMC formulation and successfully validated against physical-space fully-resolved reference solutions. The same CMC formulation is subsequently used to numerically investigate the effects of curvature for laminar flames having different effective Lewis numbers: a lean methane-air flame with $L e_{\text {eff }}=0.99$ and a lean hydrogen-air flame with $L e_{\text {eff }}=0.33$. Results suggest that curvature does not affect the conditional heat release if the effective Lewis number tends to unity, so that curvature-induced transport may be neglected. In the second part of the manuscript, the effect of turbulence on the flame morphology is qualitatively analyzed using LES-CMC simulations with and without differential diffusion for a turbulent premixed bluff body methane-air flame exhibiting local extinction behaviour. Overall, both the unity and the non-unity computations predict the characteristic "M-shaped" flame observed experimentally, although some minor differences are identified. In agreement with previous DNS studies, the findings suggest that for flames propagating in the thin reaction zone regime, turbulent mixing within the flame weakens the differential transport contribution by reducing the conditional scalar dissipation rate and accordingly the conditional diffusion of the progress variable. Future investigations should focus on a further assessment of this methodology for flames propagating in different regimes (e.g. $\mathrm{Ka}<1)$.

\section{Acknowledgements}

Financial support from the Swiss Federal Office of Energy (Grant SI/500970-01) and the Swiss Competence Centre Energy and Mobility (P-No 803 SCHE-Dual) is gratefully acknowledged. The authors further thank Dr. C. E. Frouzakis and Dr. G. Giannakopoulos for fruitful discussions.

\section{References}

[1] Y. Huang, V. Yang, Prog. Energy Combust. Sci. 35 (2009) 293-364.

[2] J. Driscoll, Prog. Energy Combust. Sci. 34 (2008) 91-134.

[3] B.S. Brewster, S.M. Cannon, J.R. Farmer, F. Meng, Prog. Energy Combust. Sci. 25 (1999) 353-385.

[4] F.T.C. Yuen, Ö.L. Gülder, Proc. Combust. Inst. 32 (2009) 1747-1754.

[5] P. Tamadonfar, Ö.L. Gülder, Combust. Flame 161 (2014) 3154-3165. 
[7] T.M. Wabel, A.W. Skiba, J.F. Driscoll, Proc. Combust. Inst. 36 (2017) 1801-1808.

[8] B. Zhou, Q. Li, Y. He, P. Petersson, Z. Li, M. Aldén, X.-S. Bai, Combust. Flame 162 (2015) 2954-2958.

[9] B. Zhou, C. Brackmann, Q. Li, Z. Wang, P. Petersson, Z. Li, M. Aldén, X. Bai, Combust. Flame 162 (2015) $2937-2953$.

[10] B. Zhou, C. Brackmann, Z. Wang, Z. Li, M. Richter, M. Aldén, X.-S. Bai, Combust. Flame (2016) in press.

[11] B. Zhou, C. Brackmann, Z. Li, M. Aldén, X.-S. Bai, Proc. Combust. Inst. 35 (2015) 1409-1416.

[12] R.S. Barlow, M.J. Dunn, M.S. Sweeney, S. Hochgreb, Combust. Flame 159 (2012) 2563-2575.

[13] O. Stein, A.M. Kempf, J. Janicka, Combust. Sci. Technol. 179 (2007) 173-189.

[14] T. Poinsot, D. Veynante, Theoretical and Numerical Combustion, RT Edwards, Inc., 2005.

[15] N. Swaminathan, K.N.C. Bray, Turbulent Premixed Flames, Cambridge University Press, 2011.

[16] S. Amzin, N. Swaminathan, J.W. Rogerson, J.H. Kent, Combust. Sci. Technol. 184 (2012) 1743-1767.

[17] S.H. Chung, J.S. Kim, C.K. Law, Symp. Combust. 21 (1988) 1845-1851.

[18] S. Candel, D. Veynante, F. Lacas, E. Maistreet, N. Darabiha, T. Poinsot, in:, B. Larrouturou (Ed.), Recent Adv. Combust. Model. 6, 1990, pp. 19-64.

[19] E.R. Hawkes, R.S. Cant, Proc. Combust. Inst. 28 (2000) 51-58.

[20] M. Herrmann, Combust. Flame 145 (2006) 357-375.

[21] K.N.C. Bray, Symp. Combust. 17 (1979) 223-233.

[22] K.N.C. Bray, in:, Turbul. React. Flows, Springer, 1980, pp. 115-183.

[23] J. Galpin, A. Naudin, L. Vervisch, C. Angelberger, O. Colin, P. Domingo, Combust. Flame 155 (2008) 247-266.

[24] P. Domingo, L. Vervisch, D. Veynante, Combust. Flame 152 (2008) 415-432.

[25] H. Pitsch, L.D. De Legeneste, Proc. Combust. Inst. 29 (2002) 2001-2008.

[26] D. Bradley, P.H. Gaskell, X.J. Gu, A. Sedaghat, Combust. Flame 143 (2005) 227-245.

[27] E.R. Hawkes, J.H. Chen, Proc. Combust. Inst. 30 (2005) 647-655.

[28] J.A. van Oijen, G.R.A. Groot, R.J.M. Bastiaans, L.P.H. de Goey, Proc. Combust. Inst. 30 (2005) 657-664.

[29] R.J.M. Bastiaans, S.M. Martin, H. Pitsch, J.A. van Oijen, L.P.H. de Goey, Comput. Sci. - ICCS 20053 (2005) $64-71$.

[30] L. Vervisch, E. Bidaux, K.N.C. Bray, W. Kollmann, Phys. Fluids 7 (1995) 2496-2503.

[31] I. Langella, N. Swaminathan, Combust. Theory Model. 20 (2016) 410-440.

[32] D.C. Haworth, Prog. Energy Combust. Sci. 36 (2010) 168-259.

[33] R.P. Lindstedt, E.M. Vaos, Combust. Flame 145 (2006) 495-511.

[34] D.H. Rowinski, S.B. Pope, Combust. Theory Model. 17 (2013) 610-656.

[35] A.Y. Klimenko, R.W. Bilger, Prog. Energy Combust. Sci. 25 (1999) 595-687.

[36] Y. Wright, G. De Paola, K. Boulouchos, E. Mastorakos, Combust. Flame 143 (2005) 402-419.

[37] H. Zhang, A. Garmory, D.E. Cavaliere, E. Mastorakos, Proc. Combust. Inst. 35 (2015) 1167-1174. 
[38] S. Amzin, N. Swaminathan, Combust. Theory Model. 17 (2013) 1125-1153.

[39] N. Swaminathan, R.W. Bilger, Combust. Theory Model. 5 (2001) 241-260.

[40] D. Farrace, K. Chung, S.S. Pandurangi, Y.M. Wright, K. Boulouchos, N. Swaminathan, Proc. Combust. Inst. 36 (2017) 1977-1985.

[41] M. Day, J. Bell, P.-T. Bremer, V. Pascucci, V. Beckner, M. Lijewski, Combust. Flame 156 (2009) 1035-1045.

[42] M. Baum, T.J. Poinsot, D.C. Haworth, N. Darabiha, J. Fluid Mech. 281 (1994) 1-32.

[43] H. Wang, K. Luo, J. Fan, Energy \& Fuels 27 (2013) 549-560.

[44] A.J. Aspden, M.S. Day, J.B. Bell, Proc. Combust. Inst. 33 (2011) 1463-1471.

[45] C. Altantzis, C.E. Frouzakis, A.G. Tomboulides, K. Boulouchos, Combust. Flame 162 (2015) 331-344.

[46] A.J. Aspden, M.S. Day, J.B. Bell, Proc. Combust. Inst. 33 (2011) 1473-1480.

[47] A.J. Aspden, M.S. Day, J.B. Bell, Combust. Flame 166 (2016) 266-283.

[48] H. Wang, E.R. Hawkes, B. Zhou, J.H. Chen, Z. Li, M. Aldén, Proc. Combust. Inst. (2016) in press.

[49] B. Savard, B. Bobbitt, G. Blanquart, Proc. Combust. Inst. 35 (2015) 1377-1384.

[50] S. Lapointe, B. Savard, G. Blanquart, Combust. Flame 162 (2015) 3341-3355.

[51] M. Matalon, C. Cui, J.K. Bechtold, J. Fluid Mech. 487 (2003) 179-210.

[52] B. Savard, G. Blanquart, Combust. Flame 161 (2014) 1547-1557.

[53] R. McDermott, S.B. Pope, J. Comput. Phys. 226 (2007) 947-993.

[54] H. Pitsch, N. Peters, Combust. Flame 114 (1998) 26-40.

[55] F. Mauss, D. Keller, N. Peters, Symp. Combust. 23 (1991) 693-698.

[56] Y. Xuan, G. Blanquart, M.E. Mueller, Combust. Flame 161 (2014) 1294-1309.

[57] A. Kronenburg, R.W. Bilger, Phys. Fluids 9 (1997) 1435-1447.

[58] S. Navarro-Martinez, S. Rigopoulos, Flow Turbul. Combust. 89 (2012) 311-328.

[59] M.-C. Ma, C.B. Devaud, Combust. Flame 162 (2015) 144-158.

[60] G. Lodier, L. Vervisch, V. Moureau, P. Domingo, Combust. Flame 158 (2011) 2009-2016.

[61] J.D. Regele, E. Knudsen, H. Pitsch, G. Blanquart, Combust. Flame 160 (2013) 240-250.

[62] E.S. Richardson, J.H. Chen, Combust. Flame 159 (2012) 2398-2414.

[63] D. Veynante, L. Vervisch, Prog. Energy Combust. Sci. 28 (2002) 193-266.

[64] A. Garmory, E. Mastorakos, Proc. Combust. Inst. 35 (2015) 1207-1214.

[65] A. Triantafyllidis, E. Mastorakos, Flow, Turbul. Combust. 84 (2010) 481-512.

[66] A. Garmory, E. Mastorakos, Int. J. Heat Fluid Flow 39 (2013) 53-63.

[67] H. Kolla, N. Swaminathan, Combust. Flame 157 (2010) 943-954.

[68] R. Kee, J. Grcar, M. Smooke, Sandia Natl. Lab. (1985) Report SAND85-8249.

[69] H.P. Tsui, M.M. Kamal, S. Hochgreb, W.K. Bushe, Combust. Flame 165 (2016) 208-222.

[70] P.A. Libby, F.A. Williams, in:, Turbul. React. Flows, Academic Press London, 1994, pp. 1-61. 
[71] S.M. Martin, J.C. Kramlich, G. Kosály, J.J. Riley, in:, Vol. 1 Turbo Expo 2002, ASME, 2002, pp. 573-580.

[72] I. Langella, N. Swaminathan, Y. Gao, N. Chakraborty, Combust. Theory Model. 19 (2015) 628-656.

[73] P. Domingo, L. Vervisch, S. Payet, R. Hauguel, Combust. Flame 143 (2005) 566-586.

[74] A.W. Vreman, J.A. van Oijen, L.P.H. de Goey, R.J.M. Bastiaans, Flow, Turbul. Combust. 82 (2009) $511-535$.

[75] V. Moureau, P. Domingo, L. Vervisch, Combust. Flame 158 (2011) 1340-1357.

[76] K. Bray, M. Champion, P. Libby, N. Swaminathan, Combust. Flame 146 (2006) 665-673.

[77] T.D. Dunstan, Y. Minamoto, N. Chakraborty, N. Swaminathan, Proc. Combust. Inst. 34 (2013) $1193-1201$.

[78] J. Kariuki, J.R. Dawson, E. Mastorakos, Combust. Flame 159 (2012) 2589-2607.

[79] J. Kariuki, A. Dowlut, R. Yuan, R. Balachandran, E. Mastorakos, Proc. Combust. Inst. 35 (2015) 1443-1450.

[80] N. Peters, Turbulent Combustion, Cambridge University Press, 2000.

[81] E. Van Kalmthout, D. Veynante, Phys. Fluids 10 (1998) 2347-2368.

[82] D.O. Lignell, J.H. Chen, P.J. Smith, T. Lu, C.K. Law, Combust. Flame 151 (2007) 2-28.

[83] R.J. Kee, F.M. Rupley, J.A. Miller, Sandia Natl. Lab. (1996).

[84] G.P. Smith, GRI-Mech 3.0 (2008) available at <http://www.me.berkeley.edu/gri_mech>.

[85] R. Sankaran, E.R. Hawkes, J.H. Chen, T. Lu, C.K. Law, Proc. Combust. Inst. 31 (2007) 1291-1298.

[86] J.K. Bechtold, M. Matalon, Combust. Flame 127 (2001) 1906-1913.

[87] X.J. Gu, M.Z. Haq, M. Lawes, R. Woolley, Combust. Flame 121 (2000) 41-58.

[88] P.N. Brown, A.C. Hindmarsh, Appl. Math. Comput. 31 (1989) 40-91.

[89] T. Lu, C.K. Law, Combust. Flame 154 (2008) 761-774.

[90] A.M. Kempf, B.J. Geurts, J.C. Oefelein, Combust. Flame 158 (2011) 2408-2419. 